\title{
Association of cyclin D1 and survivin expression with sensitivity to radiotherapy in patients with nasopharyngeal carcinoma
}

\author{
S.M. Fu' ${ }^{1}$ M.X. Xu' ${ }^{2}$ S.M. Lin ${ }^{3}$, Z. Liang ${ }^{1}$ and J.H. Cai ${ }^{1}$ \\ ${ }^{1}$ Department of Medical Examination, Hainan Provincial People's Hospital, \\ Haikou, China \\ ${ }^{2}$ College of Agriculture, Hainan University, Haikou, China \\ ${ }^{3}$ Department of Radiotherapy, Hainan Provincial People's Hospital, \\ Haikou, China \\ Corresponding author: S.M. Fu \\ E-mail: fushengmiao@126.com
}

Genet. Mol. Res. 13 (2): 3502-3509 (2014)

Received May 22, 2013

Accepted December 9, 2013

Published February 14, 2014

DOI http://dx.doi.org/10.4238/2014.February.14.6

\begin{abstract}
The association between cyclin D1 and survivin protein expressions with radiotherapy sensitivity in patients with nasopharyngeal carcinoma was investigated. Biopsy specimens of 72 patients with nasopharyngeal carcinoma were collected before the initiation of radiotherapy (49 cases were in the radiationsensitive group and 23 cases were in the radiation-insensitive group). Conventional hematoxylin and eosin staining was used for tissue typing. The immunohistochemical SP method was used to detect cyclin D1 and survivin protein expression levels. The IBM SPSS Statistics 20 statistical software was applied for conducting the chi-squared test and the Spearman correlation analysis. In the 72 cases, the high expression rates of cyclin D1 were $28.6 \%(14 / 49)$ and $69.6 \%(16 / 23)$ in the radiotherapy-sensitive group and in the radiotherapy-insensitive group, respectively, and the differences between groups were statistically significant $(\mathrm{P}<0.05)$. The high
\end{abstract}


expression rates of survivin were $34.7 \%(17 / 49)$ and $73.9 \%(17 / 23)$ in the radiotherapy-sensitive group and in the radiotherapy-insensitive group, respectively, which differed significantly $(\mathrm{P}<0.05)$. The protein expressions of cyclin D1 and survivin were positively correlated (Spearman's $r=0.353, \mathrm{P}<0.05$ ). Cyclin D1 and survivin expression levels were negatively correlated with the radiosensitivity of nasopharyngeal carcinoma. Cyclin D1 and survivin may be used as molecular markers to predict the sensitivity of radiotherapy.

Key words: Cyclin D1; Survivin; Nasopharyngeal carcinoma

\section{INTRODUCTION}

Nasopharyngeal carcinoma is a common head and neck cancer in the southern provinces of China and in Southeast Asia. Radiation therapy is the main treatment for nasopharyngeal carcinoma; however, obvious individual differences in the sensitivity to radiation therapy have been observed in patients with nasopharyngeal carcinoma in clinical practices. Therefore, the identification of radiation therapy-sensitive molecular markers for patients with nasopharyngeal carcinoma would be of great value for developing a reasonable individualized radiotherapy program. In this study, immunohistochemical techniques were used to detect the protein expression levels of cyclin D1 and survivin in radiotherapy-sensitive patients and -insensitive patients. We also explored the significance of these proteins to the radiation therapy sensitivity of nasopharyngeal carcinoma to provide a theoretical basis for the early and accurate prediction of radiotherapy sensitivity.

\section{MATERIAL AND METHODS}

\section{Clinical data}

Samples were obtained from patients visiting the Department of Otolaryngology in Hainan Provincial People's Hospital from March 2009 to September 2011, and all diagnoses were confirmed by biopsy pathology. All patients received radiotherapy in the radiology department of our hospital, and complete clinical data were available for all patients. All patients were first diagnosed patients who did not receive any anti-cancer treatment prior to enrolling in the study. Fifty-five cases were males and 17 cases were females, and subjects ranged in age from 20 to 69 years with a median age of 41 years. Nasopharyngeal carcinoma was histopathologically classified according to the 1991 International Classification Standard published by the World Health Organization (WHO). Among the specimens of the 72 cases, 5 cases were keratinizing squamous cell carcinoma and 67 cases were non-keratinizing carcinoma, while 47 cases were differentiated types and 25 cases were non-differentiated types. At the end of radiotherapy, 49 cases had completely faded away, whereas 23 cases had residual nasopharyngeal carcinoma. The clinical phasing was conducted according to the Nasopharyngeal Carcinoma 2008 Phased Program (Guangzhou, China): 7 cases were in Phase I, 13 cases were in Phase II, 31 cases were in Phase III, and 21 cases were in Phase IV. 


\section{Radiotherapy program}

The radiation therapy used the X-ray from a $6 \mathrm{MeV}$ medical linear accelerator, with 200 cGy conventional fractionated irradiation once a day, five times a week. The nasopharynx dose $\left(D_{T}\right)$ was 7000 to 7800 cGy, 35 to 39 times for 7 to 8 weeks.

\section{Efficacy evaluation}

All patients were observed for 3 months after the completion of radiation therapy and they received computed tomography scans of the nasopharynx. The cancer fade away status and the term efficacy of radiation therapy were evaluated. The efficacy evaluation was performed according to the Response Evaluation Criteria in Solid Tumors (RECIST) (2009 revision). Complete response (CR) was identified following complete disappearance of the tumor mass. A partial response (PR) was identified when the tumor mass shrunk $\geq 50 \%$. No change (NC) was identified when the tumor mass shrunk $<50 \%$, or when the tumor mass increase was $\leq 25 \%$. Progressive disease (PD) was identified when one or more mass increased by $\geq 25 \%$, or following the appearance of a new mass. The radiation efficacy was classified as sensitive in cases of CR and PR and insensitive in cases of NC and PD.

\section{Reagents}

Cyclin D1 antibody (rabbit anti-human monoclonal antibody), survivin antibody (rabbit anti-human polyclonal antibody), Immunohistochemistry Ultra Sensitive ${ }^{\mathrm{TM}}$ SP kit (goat antirabbit), antigen retrieval solution, diaminobenzidine color reagent, and hematoxylin counterstain were all purchased from the Fuzhou Maixin Bio-Technology Development Company.

\section{Methods}

The specimens were $10 \%$ formalin fixed, paraffin embedded, and serial-sectioned into continuous $4-\mu \mathrm{m}$ slices. The histological type was determined with hematoxylin and eosin staining under an optical microscope. The immunohistochemical staining was performed using the SP method, with specific steps optimally designed in accordance with the operating instructions of the kit. The positive control slides provided in the kit were used as the positive control. Phosphate-buffered solution was used as the negative control instead of primary antibody, and staining was carried out under the same conditions.

\section{Determination of results}

All immunohistochemical slices were independently counted by three pathologists under optical microscope. Each pathologist randomly selected 10 fields in each slice under $10 \times 40$ high magnification. The percentage of accurately positioned immunohistochemically positive cells of all cancer cells were counted and scored. The expression of positive cells was divided into 4 degrees: 0 to $25 \%$ positive cells was referred as "-", 26 to $50 \%$ was referred to as "+", 51 to $75 \%$ was referred as "++", and 76 to $100 \%$ was referred to as " + + +"; high expression was defined when the positive cell intensity reached "++". 


\section{Statistical analysis}

The IBM SPSS Statistics 20 statistical software was employed for all analyses. The chi-squared test and Spearman correlation analysis were used to compare data between groups, and $\mathrm{P}<0.05$ was considered to be statistically significant.

\section{RESULTS}

\section{Cyclin D1 expression in radiotherapy patients with nasopharyngeal carcinoma}

The cyclin D1 protein-positive expression site was within the cell nucleus, which presented as a brownish-yellow color. Of the 72 cases, 30 specimens had high expression, with a positive expression rate of $41.7 \%$. The high expression rates of cyclin D1 were $28.6 \%(14 / 49)$ and $69.6 \%(16 / 23)$ in the radiotherapy-sensitive group and the radiotherapy-insensitive group, respectively, and the difference was significant $(\mathrm{P}<0.05)$ (Table 1 and Figure $1 \mathrm{~A}$ and $\mathrm{B}$ ).

Table 1. Expression of cyclin D1 in nasopharyngeal carcinoma patients.

\begin{tabular}{|c|c|c|c|c|c|c|c|}
\hline \multirow[t]{2}{*}{ Effect of radiotherapy } & \multirow[t]{2}{*}{$\mathrm{N}$} & \multicolumn{2}{|c|}{ Low expression } & \multicolumn{2}{|c|}{ High expression } & \multirow[t]{2}{*}{$\chi^{2}$} & \multirow[t]{2}{*}{$\mathrm{P}$} \\
\hline & & - & + & ++ & +++ & & \\
\hline Radiotherapy-sensitive group & 49 & 21 & 14 & 10 & 4 & 12.118 & $<0.05$ \\
\hline Radiotherapy-insensitive group & 23 & 3 & 4 & 9 & 7 & & \\
\hline Total & 72 & 24 & 18 & 19 & 11 & & \\
\hline
\end{tabular}

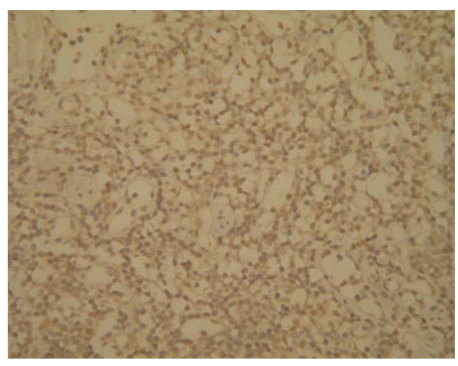

A

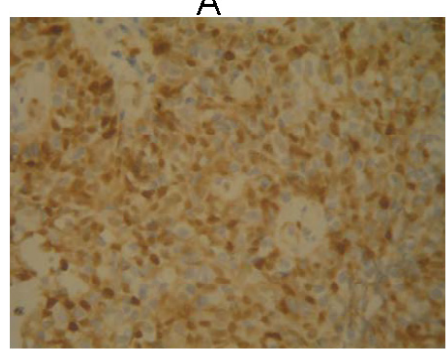

C

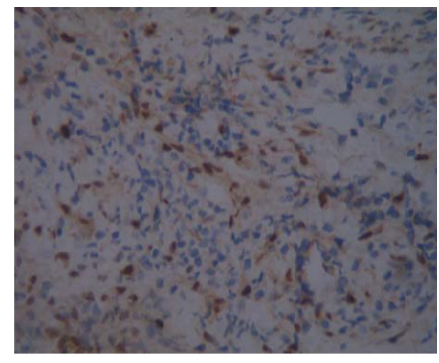

B

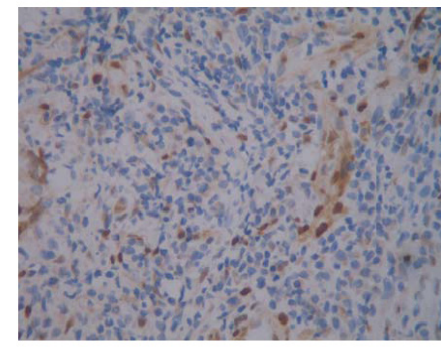

D

Figure 1. A. High expression of cyclin D1 protein in nasopharyngeal carcinoma (NPC) (diaminobenzidine, 400X); B. low expression of cyclin D1 protein in NPC; C. high expression of survivin protein in NPC; D. low expression of survivin protein in NPC. 


\section{Survivin expression in radiotherapy patients with nasopharyngeal carcinoma}

The survivin protein-positive expression site was within the cell nucleus and the cytoplasm, which presented as a brownish-yellow color. Of the 72 cases, 34 specimens had high expression, with a positive expression rate of $47.2 \%$. The high expression rates of survivin were $34.7 \%(17 / 49)$ and $73.9 \%(17 / 23)$ in the radiotherapy-sensitive group and the radiotherapy-insensitive group, respectively, and the difference was significant $(\mathrm{P}<0.05)$ (Table 2 and Figure $1 \mathrm{C}$ and $\mathrm{D})$.

\begin{tabular}{|c|c|c|c|c|c|c|c|}
\hline \multirow[t]{2}{*}{ Effect of radiotherapy } & \multirow[t]{2}{*}{$\mathrm{N}$} & \multicolumn{2}{|c|}{ Low expression } & \multicolumn{2}{|c|}{ High expression } & \multirow[t]{2}{*}{$\chi^{2}$} & \multirow[t]{2}{*}{$\mathrm{P}$} \\
\hline & & - & + & ++ & +++ & & \\
\hline Radiotherapy-sensitive group & 49 & 17 & 15 & 14 & 3 & 10.424 & $<0.05$ \\
\hline Radiotherapy-insensitive group & 23 & 3 & 3 & 12 & 5 & & \\
\hline Total & 72 & 20 & 18 & 26 & 8 & & \\
\hline
\end{tabular}

\section{Expression analysis of the cyclin D1 and survivin proteins in nasopharyngeal carcinoma patients treated with radiotherapy}

In the specimens of the 72 cases, 30 cases had high cyclin D1 expression and 42 cases had low expression. Similarly, 34 cases had high survivin expression and 38 cases had low expression. Twenty-two cases showed high expression of both proteins and 30 cases had low expression of both proteins. The protein expressions of cyclin D1 and survivin were positively correlated (Spearman $r=0.353, \mathrm{P}<0.05$ ) (Table 3).

\begin{tabular}{|c|c|c|c|c|c|}
\hline \multirow[t]{2}{*}{ Cyclin D1 } & \multicolumn{4}{|c|}{ Survivin } & \multirow[t]{2}{*}{ Total } \\
\hline & - & + & ++ & +++ & \\
\hline & 10 & 7 & 5 & 2 & 24 \\
\hline + & 4 & 9 & 4 & 1 & 18 \\
\hline ++ & 4 & 2 & 11 & 2 & 19 \\
\hline +++ & 2 & 0 & 6 & 3 & 11 \\
\hline Total & 20 & 18 & 26 & 8 & 72 \\
\hline
\end{tabular}

\section{DISCUSSION}

Currently, radiation therapy is the main treatment for nasopharyngeal carcinoma, and tumor radiation sensitivity is the key to successful tumor radiation treatment. In clinical practices, individual nasopharyngeal carcinoma patients show different radiation sensitivities. In order to improve the effects of radiotherapy in patients, to achieve early prediction of patients with different radiation sensitivities, and to develop a reasonable individualized radiotherapy program, rapid, accurate, and sensitive indicators capable of predicting individual sensitivity in patients with nasopharyngeal carcinoma radiotherapy are needed. Such markers would facilitate the development of appropriate individualized radiotherapy programs. 
Tumors result from abnormal cell cycle control caused by a class of abnormal gene expressions. The cell cycle is regulated by many factors, wherein the cyclin protein plays a particularly important role. Cyclin is an important positive regulatory factor for cell cycles, and it plays an important role in cell division and proliferation through its association with cell cycle-dependent kinase (CDK). The D-type of cyclin consists of 3 subtypes, D1, D2, and D3, which show differential expression in different cell types, and have different biological functions. Cyclin D1 was found to be the most closely associated with tumor genes involved in cell cycle regulation (Ye et al., 2005). Cyclin D1 is also known as Bcl-1, which is encoded by the CCND1 gene located on chromosome 11q13. Cyclin D1 is a nuclear protein, and combines with CDK4/6 to form the active cyclin D1/CDK4/6 complex, which is involved in the retinoblastoma protein $(\mathrm{pRb})$ phosphorylation at the G1- and S-phases of the cell cycle. This promotes cells to enter the S-phase and is considered the traveling control point of the G1/Sphase transition. Coco et al. (1999) found that cyclin D1 expression was increased by 6-fold in the breast cancer cell line MCF7 in the absence of tetracycline culture, and that cyclin D1 overexpressed cells could induce apoptosis more efficiently and were more sensitive to radiation than control cells. Ding and Li (2010) found that cyclin D1 expression levels were positively correlated with nasopharyngeal carcinoma cell radiosensitivity. However, Jirawatnotai et al. (2011) found that after the depletion of the human tumor cyclin D1 levels in mice missing $\mathrm{pRb}$, the cells became more sensitive to radiation and DNA damage factors. Milas et al. (2002) also found that the expression levels of cyclin D1 were negatively correlated with tumor radiosensitivity. These studies suggested that the cyclin D1 protein was closely related to the radiosensitivity of the tumor. However, in recent years, the relationship between cyclin D1 and radiotherapy sensitivity has been scarcely studied, and its association with nasopharyngeal carcinoma radiotherapy sensitivity is not yet clear. The results of this experiment showed that high cyclin D1 expression was significantly negatively correlated with the radiosensitivity of nasopharyngeal carcinoma, indicating that nasopharyngeal carcinoma patients with low cyclin D1 expression were more sensitive to radiation. The reason may be that when cyclin D1 is highly expressed, the G1- to S-phase is shortened, and cell proliferation is promoted, thereby leading to uncontrolled cell proliferation, which promotes tumor growth. When exposed to serious endogenous oncogene-induced DNA damage and damage induced by genotoxic therapies, cyclin D1 could help the DNA repair process, which may increase the survival of cancer cells and increase the tumors' resistance to treatment. Therefore, cyclin D1 can be used as a marker in radiosensitivity evaluations for patients with nasopharyngeal carcinoma.

The radiation-induced apoptosis of tumor cells is one of the main mechanisms of radiotherapy, and the degree of tumor cell apoptosis is proportional to its radiosensitivity. Many studies have shown that targeting interference therapy in the apoptotic pathway could significantly improve the radiosensitivity of tumor cells (Eckelman et al., 2006). Apoptosis is regulated by several apoptosis-related genes. The survivin gene is an apoptosis suppressor gene that is located on chromosome $17 \mathrm{q} 25$, and its protein is a member of the inhibitor of apoptosis protein family. Survivin has dual functions in apoptosis inhibition and cell division regulation. Survivin expression is cyclical; it shows $\mathrm{G}_{2} / \mathrm{M}$-phase-specific expression, and it is expressed in the spindle to regulate cell mitosis. Survivin inhibits apoptosis through direct or indirect combinations with caspase apoptosis effector molecules, by blocking interactions with CDK2 or CDK4, and by blocking the apoptosis signal transduction pathway. Survivin is expressed in embryonic development organizations and in most of the tumor tissues examined 
in humans and other mammals to date; however, it is not expressed in normal adult tissues that have already differentiated and matured (Tanaka et al., 2000). Jin et al. (2009) used RNA interference technology to inhibit the expression of survivin in liver cancer HepG2 cell lines, which showed significant improvement in radiation sensitivity to high-linear energy transfer radiation. Lei et al. (2010) also found that when survivin expression was inhibited in renal cell carcinoma cell lines, the radiation sensitivity of the cells increased. Huang et al. (2006) used large cell sampled high-flux tissue microarray technology, and found a significantly positive correlation between survivin expression and radiation sensitivity in nasopharyngeal cancer patients. Similarly, the results of the present experiment showed that survivin expression was significantly higher in the radiotherapy-insensitive group than in the radiation-sensitive group, indicating that high survivin expression was positively correlated with resistance to radiotherapy in nasopharyngeal carcinoma. Survivin overexpression inhibits the tumor cell apoptosis pathway, which increases resistance to radiotherapy. The positive expression of survivin was mainly observed in the nucleus, which may be associated with the radiation resistance. Survivin is therefore an important indicator that can be used to reflect radiosensitivity and predict the effects of radiotherapy.

In this experiment, the cyclin D1 and survivin protein expressions were positively correlated $(r=0.353, \mathrm{P}<0.05)$. Conway et al. (2000) found that the high expression of survivin could increase the expression of cyclin D1 protein levels. Shan et al. (2009) confirmed that the inhibition of cyclin D1 expression induced G1 cell cycle arrest, which consequently reduced the survivin expression levels. One possible reason for this is that survivin might inhibit apoptosis, which would cause cyclin D1 overexpression, thereby affecting the cell cycle as described above, so that the cells would continue to proliferate leading to tumorigenesis.

Radiation therapy is an important method for cancer treatment. Its main mechanism involves damage to DNA and cell cycle changes to affect cell proliferation, eventually causing the death and apoptosis of tumor cells. Cyclin D1 and survivin may play roles in these processes, which can result in the resistance of tumor cells to radiation. The mechanisms of cyclin D1 and survivin in radiation resistance are not yet well elucidated and therefore need further study. Cyclin D1 and survivin can be considered as radiotherapy-sensitive molecular markers for patients with nasopharyngeal carcinoma to provide a theoretical basis for clinical radiation therapy and to develop individualized treatment programs. Furthermore, cyclin D1 or survivin can also be considered as potential targets to design radiotherapy to specifically inhibit their expressions and achieve better radiotherapy-sensitizer effects.

\section{ACKNOWLEDGMENTS}

Research supported by the Hainan Province Nature Science Foundation of China (\#309086).

\section{REFERENCES}

Coco Martin JM, Balkenende A, Verschoor T, Lallemand F, et al. (1999). Cyclin D1 overexpression enhances radiationinduced apoptosis and radiosensitivity in a breast tumor cell line. Cancer Res. 59: 1134-1140.

Conway EM, Pollefeyt S, Cornelissen J, DeBaere I, et al. (2000). Three differentially expressed survivin cDNA variants encode proteins with distinct antiapoptotic functions. Blood 95: 1435-1442.

Ding SL and Li G (2010). Survivin, Cyclin D1 and nm23-H1 protein expression in the relationship of the degree of 
radiotherapy for nasopharyngeal carcinoma prognosis. Shen Yang: Zhong Guo Yi Ke Da Xue 42-47.

Eckelman BP, Salvesen GS and Scott FL (2006). Human inhibitor of apoptosis proteins: why XIAP is the black sheep of the family. EMBO Rep. 7: 988-994.

Huang DH, Tian YQ and Qiu YZ (2006). Application PCNA, and survivin forecast radiotherapy sensitivity. Tong $J i D a$ Xиe Xue Bao (Yi Xue Ban) 25: 39-42.

Jin XD, Li Q, Li P, Wu QF, et al. (2009). Inhibiting Survivin Expression Increases the Radiosensitivity of Human Hepatoma HepG2 Cells to High-LET Carbon Ions. World Congr. Med. Phys. Biomed. Eng. 25: 166-169.

Jirawatnotai S, Hu Y, Michowski W, Elias JE, et al. (2011). A function for cyclin D1 in DNA repair uncovered by protein interactome analyses in human cancers. Nature 474: 230-234.

Lei Y, Geng Z, Guo-Jun W, He W, et al. (2010). Prognostic significance of survivin expression in renal cell cancer and its correlation with radioresistance. Mol. Cell Biochem. 344: 23-31.

Milas L, Akimoto T, Hunter NR, Mason KA, et al. (2002). Relationship between cyclin D1 expression and poor radioresponse of murine carcinomas. Int. J. Radiat. Oncol. Biol. Phys. 52: 514-521.

Shan BE, Wang MX and Li RQ (2009). Quercetin inhibit human SW480 colon cancer growth in association with inhibition of cyclin D1 and survivin expression through Wnt/beta-catenin signaling pathway. Cancer Invest. 27: 604-612.

Tanaka K, Iwamoto S, Gon G, Nohara T, et al. (2000). Expression of survivin and its relationship to loss of apoptosis in breast carcinomas. Clin. Cancer Res. 6: 127-134.

Ye YS, Zhan RY and Tong Y (2005). Cyclin D1 in brain glioma expression and cell cycle expression patterns of research. Zhe Jiang Lin Chuang Yi Xue 7: 899-900. 